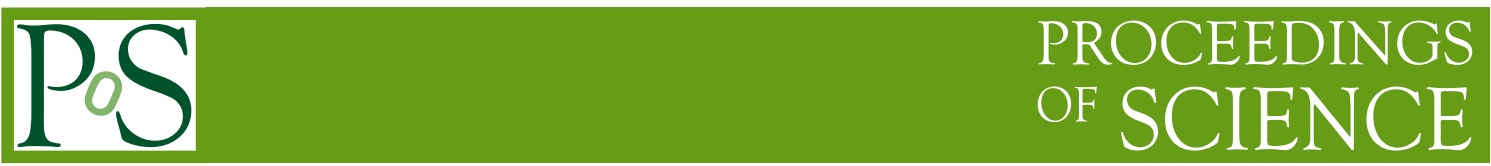

\title{
Investigation of the proton structure at $\bar{P}$ ANDA-FAIR
}

\author{
Alaa Dbeyssi* \\ Helmholtz-Institut Mainz \\ E-mail: adbeyssi@uni-mainz.de \\ Iris Zimmermann \\ Helmholtz-Institut Mainz \\ E-mail: irzimmereuni-mainz.de \\ Dmitry Khaneft \\ Helmholtz-Institut Mainz \\ E-mail: dmkhanef@uni-mainz.de
}

\section{On behalf of the $\bar{P}$ ANDA Collaboration}

\begin{abstract}
An important goal of the future PANDA Experiment at FAIR (Darmstadt, Germany) is the investigation of the proton structure. Electromagnetic proton form factors parameterize the electric and magnetic structure of protons. They can be experimentally accessed in the time-like region through the annihilation processes $\bar{p} p \rightarrow \ell^{+} \ell^{-}(\ell=e, \mu)$, assuming that the interaction takes place through the exchange of one virtual photon. The expected statistical precision for the measurement of proton form factors with $\overline{\mathrm{P}} \mathrm{ANDA}$ for the process $\bar{p} p \rightarrow \ell^{+} \ell^{-}(\ell=e, \mu)$ was investigated in the framework of the PANDARoot software for detector simulation and event reconstruction. The signal identification and the suppression of the main background process $\left(\bar{p} p \rightarrow \pi^{+} \pi^{-}\right)$is studied. Different methods have been used to generate and analyse the processes of interest. The results show that time-like electromagnetic proton form factors can be measured at $\overline{\mathrm{P}} \mathrm{ANDA}$ with high statistical accuracy over a large kinematical region.
\end{abstract}

XVII International Conference on Hadron Spectroscopy and Structure - Hadron2017

25-29 September, 2017

University of Salamanca, Salamanca, Spain

\footnotetext{
* Speaker.
} 


\section{Introduction}

The understanding of the hadron structure is one of the most challenging issues of the strong interaction in the non perturbative regime. Structure observables, such as the electromagnetic form factors, can provide essential information on the internal structure and the static properties of the hadrons. Among them, electromagnetic form factors are fundamental quantities which describe the distribution of the electric charges and magnetization within the hadrons. The number of electromagnetic form factors is determined by the spin of the hadron taking into account the symmetry properties of the electromagnetic interaction. The proton, which is a spin- $1 / 2$ particle, is characterized by the electric $G_{E}$ and the magnetic $G_{M}$ form factor. The cross section of the unpolarized elastic electron-proton scattering has been studied since the 1960s with increasing accuracy to determine the electromagnetic form factors of the proton in the region of negative 4-momentum transfer squared $\left(q^{2}<0\right.$, space-like region) [1]. However, the polarization transfer method [3] that was used for the first time in 1998, opened new questions in the field. The ratio of the electric and the magnetic form factor measured by the JLab-GEp collaboration (see [2] and references therein), based on the polarization transfer method, decreases almost linearly from unity when the momentum transfer squared increases. This result is in conflict with previous measurements. In addition to the elastic electron-proton scattering, the annihilation reactions allow to extend the form factor results from elastic electron-proton scattering into the time-like region of positive squared 4-momentum transfer squared $\left(q^{2}>0\right.$, time-like region). In the time-like region, electromagnetic form factors can be related to the time evolution of the distributions of the electric charges and magnetization within the proton [4]. In this region, proton electromagnetic form factors are accessible through measurements of differential and total cross sections of $e^{+} e^{-} \leftrightarrow \bar{p} p$, assuming that the interaction proceeds through the exchange of one virtual photon which carries the squared momentum transfer $q^{2}$. The precision of the time-like proton form factor measurements has been limited up to now by the statistical quality of the experimental data.

In this contribution, we report on feasibility studies for future measurements of the proton electromagnetic form factors in the time-like region with the PANDA (antiProton ANnihilations at DArmstadt) experiment [5] at FAIR (Facility for Antiproton and Ion Research). The future $\bar{P} A N D A$ experiment will offer unique possibilities for new investigations of the nucleon structure complementing the results obtained from the studies of lepton beam induced reactions. The proton form factors can be accessed at the $\overline{\mathrm{P}}$ ANDA experiment via the $\bar{p} p \rightarrow l^{+} l^{-}$annihilation process with $l=e, \mu$. The differential cross section for the $\bar{p} p \rightarrow l^{+} l^{-}$channel, for the case of unpolarized particles [6], can be written as:

$$
\frac{d \sigma}{d \cos \theta}=\frac{\alpha^{2} \pi(\hbar c)^{2}}{2 s} \frac{\beta_{l}}{\beta_{\bar{p}}}\left[\frac{s}{4 M_{p}^{2}}\left(1-\beta_{l}^{2} \cos ^{2} \theta\right)\left|G_{E}\right|^{2}+\left(1+\frac{s}{4 M_{l}^{2}}+\beta_{l}^{2} \cos ^{2} \theta\right)\left|G_{M}\right|^{2}\right]
$$

where $\theta$ is the angle of the outgoing lepton with negative charge in the $\bar{p} p$ center of mass frame, $\alpha \approx 1 / 137$ is the fine structure constant and $\beta_{l, p}=\sqrt{1-4 M_{l, p}^{2} / s}$ is the velocity of the lepton or the proton.

In proton-antiproton annihilation, the selection of a pair of leptons accompanied by a neutral pion in the final state allows the measurements of the proton form factors in the unphysical region, 
below the $\left(2 M_{p}\right)^{2}$ kinematic threshold [7, 8]. The time-like unphysical region has never been experimentally explored so far. The antiproton-proton annihilation into lepton pair and a neutral pion can be also investigated at the PANDA experiment [9].

\section{The $\bar{P} A N D A$ experiment}

The $\bar{P} A N D A$ experiment at FAIR will detect the products of the annihilation reactions induced by a high-intensity antiproton beam with momenta from 1.5 to $15 \mathrm{GeV} / \mathrm{c}$. The different components of the detector were designed following the experience gained in high energy experiments. The PANDA detector will be installed at the High Energy Storage Ring (HESR) of FAIR. The physics program of the PANDA experiment [5] includes charmonium spectroscopy, search for hybrids and glueballs, search for charm and strangeness in nuclei, baryon spectroscopy and hyperon physics, as well as nucleon structure studies. The $\bar{P}$ ANDA experiment will exploit this broad physics program thanks to its large detector acceptance, high spatial and momentum resolution and tracking capabilities, as well as excellent neutral and charged particle identification capabilities in a high rate environment.

\section{Feasibility study of $\bar{p} p \rightarrow e^{+} e^{-}$for the measurements of electromagnetic proton form factors at $\bar{P}$ ANDA}

Feasibility studies for the measurement of the process $\bar{p} p \rightarrow e^{+} e^{-}$at $\overline{\mathrm{P} A N D A}$ [10] have been performed through the PANDARoot software [11]. PANDARoot is the offline software for the $\overline{\mathrm{P} A N D A}$ detector simulation and event reconstruction. Full simulations for the processes $\bar{p} p \rightarrow e^{+} e^{-}$were carried out at different center-of-momentum energies between 5.4 and $13.9 \mathrm{GeV}^{2}$. A total of 300 million events of the main background process $\bar{p} p \rightarrow \pi^{+} \pi^{-}$were generated and reconstructed at three values of the center-of-momentum energy $s=5.4,8.2$ and $13.9 \mathrm{GeV}^{2}$. In order to separate the signal from the background the raw output and the particle identification (PID) probability algorithms for different sub-detectors as the ElectroMagnetic Calorimeter, the Straw Tube Tracker, the Micro Vertex Detector and Cherenkov detectors have been used. A background suppression factor of the order of $\sim 10^{8}$ was achieved, keeping at the same time a large and sufficient signal efficiency between $40 \%$ and $50 \%$, for the proton form factor measurements at $\bar{P}$ ANDA. Taking into account the ratio of cross sections $\sigma\left(\bar{p} p \rightarrow \pi^{+} \pi^{-}\right) / \sigma\left(\bar{p} p \rightarrow e^{+} e^{-}\right) \simeq 10^{6}[12,13]$, in order to make a reliable measurement of the proton form factors at $\bar{P}$ ANDA, a background rejection factor on the order of $10^{8}$ is needed in order to reduce the signal pollution to values smaller than $1 \%$. Two independent simulation studies have been performed for the signal reaction, denoted by Method I and Method II. The differences between them are (i) the angular distribution model used as input for the signal event generator ii) the number of the generated events, iii) the selection conditions and iv) the fit functions used to extract the proton electromagnetic form factors from the angular distribution of the produced lepton.

The results from the two simulations, assuming $R=\left|G_{E}\right| /\left|G_{M}\right|=1$, are shown in Fig. 1, together with the existing experimental data. The generated statistics correspond to an integrated luminosity of $2 \mathrm{fb}^{-1}$ per beam momentum setting. 


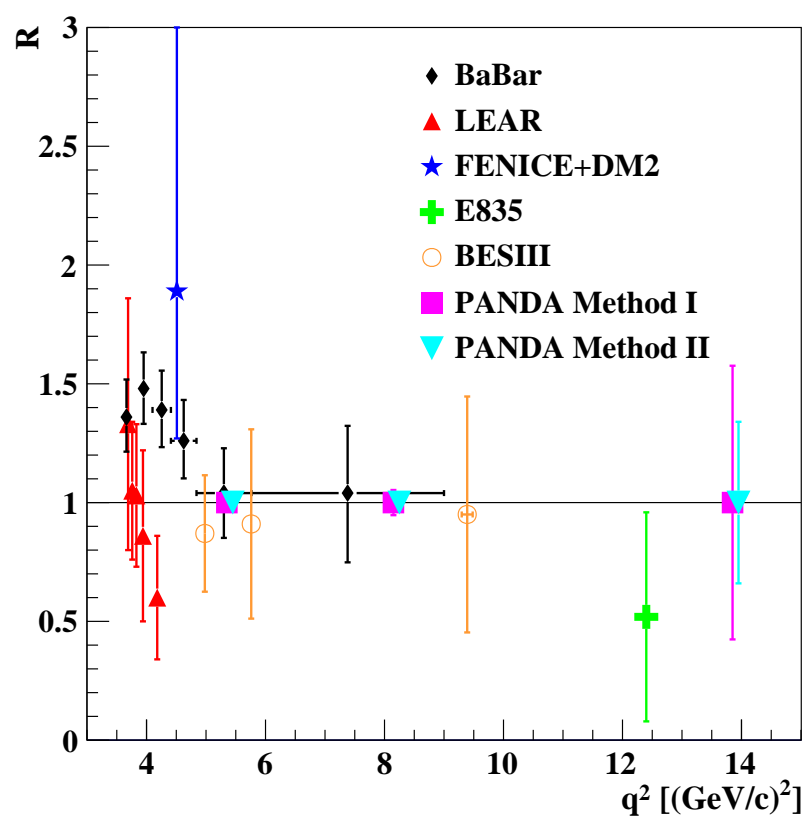

Figure 1: Expected statistical precision of the determination of the proton form factor ratio $R=\left|G_{E}\right| /\left|G_{M}\right|$ at $\overline{\mathrm{P}} \mathrm{ANDA}$ as a function of $q^{2}$, compared with the existing data ((see [10] and references therein)).

The study of the systematic uncertainties shows that the background misidentification and luminosity uncertainty dominate the total uncertainty at lower $q^{2}$, while in the high energy domain the total uncertainty is dominated by the statistical fluctuations due to the smaller $\bar{p} p \rightarrow e^{+} e^{-}$ cross section. The total (statistical and systematic) relative uncertainty is expected to be in the range $3.3-57.0 \%$ for the proton form factor ratio $R$. In addition, the extraction of individual values of $\left|G_{E}\right|$ and $\left|G_{M}\right|$ is also possible in the momentum range below $13.9(\mathrm{GeV} / c)^{2}$ with a total precision of $2.2 \%-48.0 \%$ and $3.5 \%-9.7 \%$, respectively. The absolute cross section measurement depends essentially on the precision achieved in the luminosity measurement, which is expected to be around $4 \%$. The measurement of the total cross section of $\bar{p} p \rightarrow e^{+} e^{-}$can be extended to higher values of $q^{2}$ depending on the total reconstruction efficiency for the signal reaction while a signal pollution less than $1 \%$ is achieved.

\section{Feasibility study of the $\bar{p} p \rightarrow \mu^{+} \mu^{-}$reaction for the measurements of electromagnetic proton form factors at $\bar{P} A N D A$}

The possibility to use the reaction $\bar{p} p \rightarrow \mu^{+} \mu^{-}$for the determination of the time-like electromagnetic proton form factors was studied with a MC simulation for an antiproton momentum of $p=1.7 \mathrm{GeV} / \mathrm{c}\left(\mathrm{s}=5.4 \mathrm{GeV}^{2}\right)$. This channel contains the same information about the proton form factors as the electron pair production and therefore also serves as a consistency check. One advantage of measuring $\bar{p} p \rightarrow \mu^{+} \mu^{-}$is that radiative corrections due to final state photon emission are suppressed by the muon mass and can be neglected. However in this case, the suppression of the strong hadronic background is more challenging. The muon identification is mainly based on detector information from the Muon System, while other sub-detectors show less separation 
power, which complicates the separation of the signal from the background considerably. For both the signal and the main background channel, $\bar{p} p \rightarrow \pi^{+} \pi^{-}$, two statistically independent data samples were generated. After the MC event generation, the events produced by the MC generator undergo the same standard chain of simulation and analysis as for $\bar{p} p \rightarrow e^{+} e^{-}$. A method based on multivariate data classification was used to optimize the separation of the signal from the background (Boosted Decision Trees). A signal to background ratio of 1:4 (background rejection factor of $\sim 10^{-6}$ ) was achieved. Due to the insufficient background rejection, a subtraction of residual background was applied in the analysis. In order to study the effect of the statistical background fluctuations on the precision of the form factor measurements, the two statistically independent MC samples of $10^{8}$ events each for $\bar{p} p \rightarrow \pi^{+} \pi^{-}$, have been used. After the full data selection, one of the samples was added to the signal and the second sample was used for the background subtraction. An efficiency correction follows the background subtraction using the reconstruction efficiency distribution of the signal. After the background subtraction, the reconstructed signal events were corrected using the signal reconstruction efficiency. The form factor ratio $R,\left|G_{E}\right|$ and $\left|G_{M}\right|$ were extracted from fitting the efficiency corrected angular distribution $\bar{p} p \rightarrow \mu^{+} \mu^{-}$. At 1.7 $\mathrm{GeV} / \mathrm{c}$, a statistical precision on $R$ of $\sim 5.1 \%$ (Fig. 2), on $\left|G_{E}\right|$ of $\sim 8.6 \%$ and on $\left|G_{M}\right|$ of $\sim 4.1 \%$ were obtained. The feasibility studies of the $\bar{p} p \rightarrow \mu^{+} \mu^{-}$reaction will be extended to other beam momenta like $1.5,2.5$ and $3.3 \mathrm{GeV} / \mathrm{c}$.

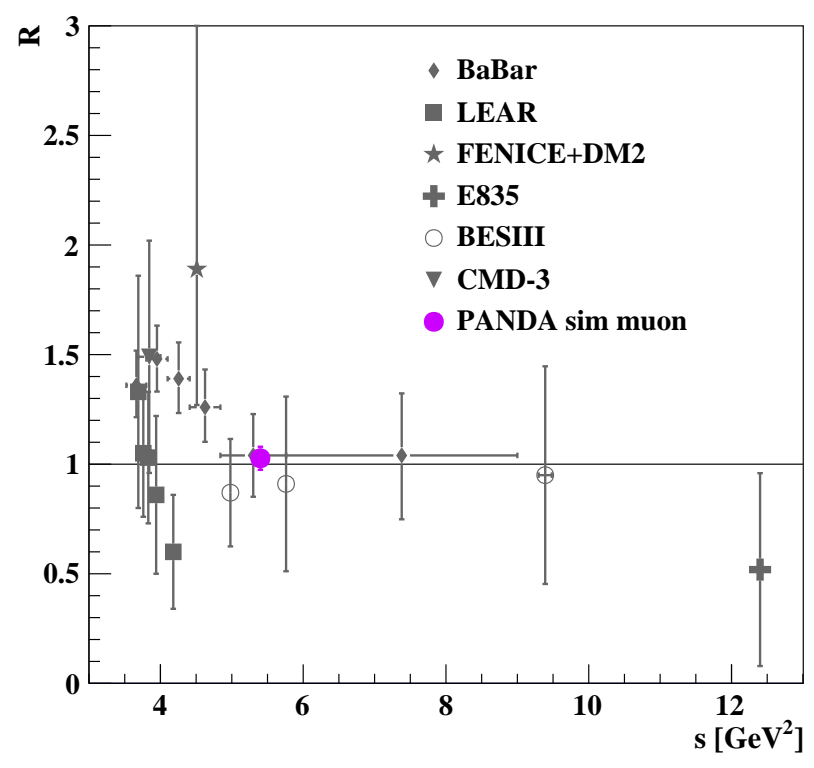

Figure 2: Expected statistical precision of the determination of the proton form factor ratio $R=\left|G_{E}\right| /\left|G_{M}\right|$ at $\overline{\mathrm{P}}$ ANDA from the study of $\bar{p} p \rightarrow \mu^{+} \mu^{-}$in comparison to existing world data.

\section{Conclusion}

The $\bar{P}$ ANDA experiment at FAIR will extend the knowledge of the time-like electromagnetic proton form factors in a large kinematic range. Feasibility studies for the measurement of the processes $\bar{p} p \rightarrow \ell^{+} \ell^{-}(\ell=e, \mu)$ have been performed. The results of the simulations show that 
the $\left|G_{E}\right|,\left|G_{M}\right|$ proton form factors and their ratio will be measureable at PANDA up to $q^{2}=13.9$ $(\mathrm{GeV} / \mathrm{c})^{2}$ with a high precision. It will be the first time that muons in the final state will be used to measure the time-like form factors of the proton. The possibility to access the relative phase of the proton time-like form factors using a transversally polarized proton target at PANDA will also be investigated. The $\bar{P} A N D A$ experiment will provide a complementary study of the nucleon structure with the annihilation processes; so, future data from the $\bar{P} A N D A$ experiment are expected to be able to set new stringent tests of nucleon models.

\section{References}

[1] C. F. Perdrisat, V. Punjabi and M. Vanderhaeghen, Nucleon Electromagnetic Form Factors, Prog. Part. Nucl. Phys. 59, 694 (2007) [hep-ph/ 0612014$].$

[2] A. J. R. Puckett et al., Recoil Polarization Measurements of the Proton Electromagnetic Form Factor Ratio to $Q^{2}=8.5 \mathrm{GeV}^{2}$, Phys. Rev. Lett. 104, 242301 (2010) [arXiv:1005.3419 [nucl-ex]].

[3] A. I. Akhiezer and M. P. Rekalo, Polarization phenomena in electron scattering by protons in the high energy region, Sov. Phys. Dokl. 13, 572 (1968) [ Dokl. Akad. Nauk Ser. Fiz. 180, 1081 (1968)].

[4] E. A. Kuraev, E. Tomasi-Gustafsson and A. Dbeyssi, A Model for space and time-like proton (neutron) form factors, Phys. Lett. B 712, 240 (2012) [arXiv:1106.1670 [hep-ph] ].

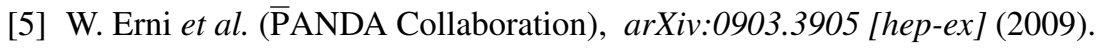

[6] A. Zichichi, S. M. Berman, N. Cabibbo and R. Gatto, Proton anti-proton annihilation into electrons, muons and vector bosons, Nuovo Cim. 24, 170 (1962).

[7] C. Adamuscin, E. A. Kuraev, E. Tomasi-Gustafsson and F. E. Maas, Testing axial and electromagnetic nucleon form factors in time-like regions in the processes $\bar{p}+n \rightarrow \pi^{-}+\ell^{-}+\ell^{+}$and $\bar{p}+p \rightarrow \pi^{0}+\ell^{-}+\ell^{+}, \ell=e, \mu$, Phys. Rev. C 75, 045205 (2007) [hep-ph/ 0610429 ].

[8] J. Guttmann and M. Vanderhaeghen, Theoretical Analysis of the $p \bar{p} \rightarrow \pi^{0} e^{+} e^{-}$Process within a Regge Framework, Phys. Lett. B 719, 136 (2013) [arXiv:1210.3290 [hep-ph] ].

[9] J. Boucher, Feasibility studies of the $\bar{p} p \rightarrow e^{+} e^{-} \pi^{0}$ electromagnetic channel at $\overline{\mathrm{P}} A N D A, P h D$ thesis, University of Paris-Sud XI and of the Johannes Gutenberg University, 2011.

[10] B. Singh et al. ( $\overline{\mathrm{P} A N D A}$ Collaboration), Feasibility studies of time-like proton electromagnetic form factors at $\overline{\mathrm{P} A N D A}$ at FAIR, Eur. Phys. J. A 52, no. 10, 325 (2016) [arXiv: 1606.01118 [hep-ex]].

[11] S. Spataro, Event Reconstruction in the PandaRoot framework, Journal of Physics: Conference Series, 396 (2) 022048 (2012).

[12] J. Van de Wiele and S. Ong, Regge description of two pseudoscalar meson production in antiproton-proton annihilation, Eur. Phys. J. A 46, 291 (2010) [ arXiv:1004.2152 [nucl-th]].

[13] Y. Wang, Y. M. Bystritskiy and E. Tomasi-Gustafsson, Antiproton-proton annihilation into charged light meson pairs within effective meson theory, Phys. Rev. C 95, no. 4, 045202 (2017) [arXiv:1512.05520 [hep-ph]]. 